\title{
Complexity Analysis of Time-Frequency Features for Vibration Signals of Rolling Bearings Based on Local Frequency
}

\author{
Youfu Tang $\mathbb{D}^{1}{ }^{1}$ Feng Lin, ${ }^{1}$ and Qian Zou ${ }^{2}$ \\ ${ }^{1}$ School of Mechanical Science and Engineering, Northeast Petroleum University, Daqing 163318, China \\ ${ }^{2}$ Research Institute of Petroleum Exploration and Development, Beijing 100083, China \\ Correspondence should be addressed to Youfu Tang; tang_youfu210@163.com
}

Received 30 March 2019; Revised 9 June 2019; Accepted 18 June 2019; Published 10 July 2019

Academic Editor: Franck Poisson

Copyright (c) 2019 Youfu Tang et al. This is an open access article distributed under the Creative Commons Attribution License, which permits unrestricted use, distribution, and reproduction in any medium, provided the original work is properly cited.

The multisource impact signal of rolling bearings often represents nonlinear and nonstationary characteristics, and quantitative description of the complexity of the signal with traditional spectrum analysis methods is difficult to be obtained. In this study, firstly, a novel concept of local frequency is defined to develop the limitation of traditional frequency. Then, an adaptive waveform decomposition method is proposed to extract the time-frequency features of nonstationary signals with multicomponents. Finally, the normalized Lempel-Ziv complexity method is applied to quantitatively measure the time-frequency features of vibration signals of rolling bearings. The results indicate that the time-frequency features extracted by the proposed method have clear physical meanings and can accurately distinguish the different fault states of rolling bearings. Furthermore, the normalized Lempel-Ziv complexity method can quantitatively measure the nonlinearity of the multisource impact signal. So, it supplies an effective basis for fault diagnosis of rolling bearings.

\section{Introduction}

Rolling bearings play an important role in rotating machinery. However, due to their loud sound, poor damping, and shock resistance features, the failure rate is higher compared with other parts. Scholars home and abroad have paid more attention on healthy monitoring and fault diagnosis of rolling bearings [1-4]. Particularly, under the long-term and highspeed operation, the rolling bearings may easily produce some different faults, such as peeling, abrasion, and gluing. As is known to all, in the field of mechanical fault diagnosis, the feature extraction method of vibration signal is continuously improved with the development of signal processing technology. Various advanced fault feature extraction methods have been extensively studied [5-8]. For the vibration signal of rolling bearings, it usually presents multisource impact characteristics, which are typically nonlinear and nonstationary $[9,10]$. Because of the special mapping relationship between the excitation of equipment components and the frequency of vibration signals, the focus of mechanical fault diagnosis research is mainly on spectral analysis and timefrequency analysis $[11,12]$.
At present, there are two frequency concepts that are well known. One is the traditional frequency defined for harmonic signals [13], and the other is the instantaneous frequency defined for the narrowband signal [14]. For a long time, the concept of frequency has only a clear physical meaning for the dynamic signal of a simple harmonic vibration. Since Joseph Fourier proposed the mathematical expression of the Fourier transform, the traditional frequency obtained a perfect physical interpretation in the feature extraction of both the periodic and nonperiodic signals. But the Fourier transform can only obtain information on which harmonic frequency components and relative intensity information of each component are contained and cannot obtain information on how these frequency components evolve with time. Therefore, it is the most effective tool for analyzing and processing the stationary signal. When the signal is nonstationary, the feature extracted by the Fourier transform only measures the degree of similarity between the signal and the harmonic basis function, rather than the actual frequency information of the vibration signal [15]. The concept of instantaneous frequency can effectively measure the time-varying features of 
nonstationary signals. It has been successfully applied in the fields of radar sonar detection, seismic monitoring, electronic communication, and mechanical equipment fault diagnosis [16, 17]. However, the instantaneous frequency has only a clear physical meaning for the narrowband signal. It is considered that the narrowband signal has only one frequency component at each instantaneous time point, and much large-scale frequency information is lost. Even for many nonstationary signals, there is lack of clear physical meaning and even cannot be calculated.

In the past few decades, a series of theories and techniques of adaptive signal decomposition and transient feature extraction have been developed and widely used in various disciplines and engineering fields (e.g., Fourier transform [18], short-time Fourier transform [19], wavelet transform [20], second-generation wavelet transform [21], multiwavelet transform [22], chirplet transform [23], and atomic decomposition [24]). All of them are based on the basic function expansion of the inner product transform principle, performing the similarity measure between the signal and the basis function [25]. However, for the rolling bearings, the vibration signal represents strong nonlinearity, nonstationarity, and multisource impact, so it is unlikely to adapt all waveform features using one or several given basis functions. In the absence of sufficient prior knowledge, if a fixed basis function is employed for signal decomposition, erroneous information will be generated and the physical meaning will be unclear. Feature information sufficient to identify the fault is difficult to extract. In recent years, some adaptive signal decomposition methods, including empirical mode decomposition (EMD) [26], local mean decomposition (LMD) [27] and local characteristic-scale decomposition (LCD) [28], wavelet modulus maxima, and synchronous detection [29], improved orthogonal matching pursuit and K-SVD algorithm with adaptive transient dictionary [30], have been gradually proposed. Those methods are not required to predict the feature information contained in the analyzed signal, and they completely follow the waveform of the signal. Thus, they exhibit good adaptability in signal processing.

Although many related achievements have been achieved in the field, they also have the following problems: (1) The magnitude of the feature amplitudes under different states is greatly different, the real physical meaning is ambiguous, and the comparability is poor. (2) The feature amplitude under the same state may be fluctuated greatly with different samples, so the repeatability and stability of the results are poor. (3) The effects of noise interference are different to the accuracy of the results. Those problems are closely related to the nonlinear dynamic characteristics of rolling bearings. How to judge and quantitatively describe the nonlinearity and evolution of rolling bearings under different states is an important prerequisite for fault diagnosis. In the field of nonlinearity, some quantitative indicators including Lyapunov exponents [31], correlation dimension [32], entropy [33], and complexity [34] have been well applied. Among of them, the Lempel-Ziv complexity (LZC) has many good advantages to measure the time series arose from the nonlinear dynamics system, which have been used widely in data compression [35], coding [36], generation of test signals [37], and so on. Additionally, compared with the methods of spectral analysis and time-frequency analysis, the LZC method can detect the long-range correlations embedded in the seemingly nonstationary time series and also avoid the spurious detection of apparent long-range correlations that are an artifact of nonstationarity [38].

In order to improve the diagnosis accuracy of rolling bearings, effective and quantitative features should be extracted firstly. On the one hand, the vibration signal of rolling bearings represents the complex feature of nonlinearity and nonstationarity, the typical and useful feature information may be not significant. On the other hand, the vibration signal of rolling bearings may be strongly influenced by background noise. For solving the above problems, an integration approach based on adaptive waveform decomposition (AWD) and Lempel-Ziv complexity (LZC) was proposed. Using the AWD method, the nonstationary multicomponent signal of rolling bearings can be converted into a series of stationary single component signal which are rich in useful feature information. The LZC method is applied to quantitatively describe the complexity of nonlinear signal in the time-frequency domain. Therefore, both the AWD method and LZC algorithm have their own function in improving the diagnosis accuracy of rolling bearings, and the integration methods of AWD and LZC will have the best effect.

\section{Time-Frequency Feature Extraction Based on Local Frequency and Adaptive Waveform Decomposition}

2.1. Definition of Local Frequency. For an arbitrary signal $x(t)$, assume that the corresponding discrete time series is $\{x(i) \mid i=1,2,3, \ldots, n\}$. The time domain waveform of the signal $x(t)$ is shown in Figure 1, and the local maximum of the $x(t)$ satisfies the following equation:

$$
\begin{aligned}
x(i) & \geq x(i-1), \\
x(i) & \geq x(i+1), \\
x(i-1) & \neq x(i+1) .
\end{aligned}
$$

From the minimum waveform required to complete a vibration, the original signal can be approximated as consisting of a series of $\mathrm{V}$ waves containing two adjacent maxima, as shown in Figure 2.

Assuming that the $\mathrm{V}$ wave start position $t_{k}$ is the time at which the $k$ th local maximum value in the original signal $x(t)$ is located and $k=1,2,3, \cdots, N$, according to the $\mathrm{V}$ wave, the local period $T(t)$ of the original signal $x(t)$ can be defined as follows:

$$
T(t)=t_{k+1}-t_{k}, \quad t_{k} \leq t<t_{k+1},
$$

where $T(t)$ represents the time required for the signal to complete a complete local vibration over a local time range.

Similarly, a local mean $m(t)$ and a local amplitude $a(t)$ are also given below: 


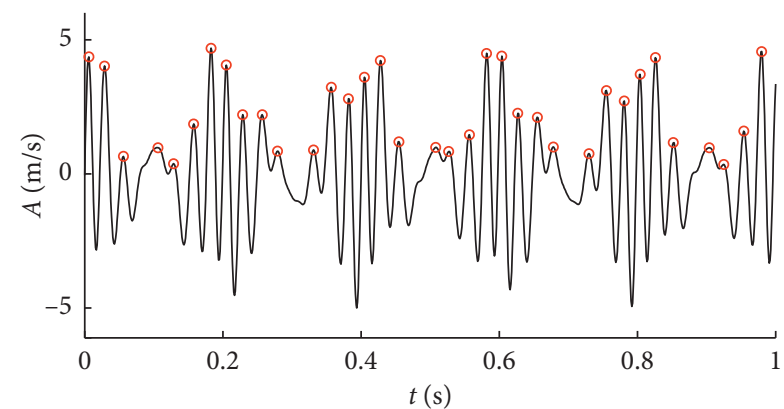

Figure 1: The time domain waveform of the signal $x(t)$.

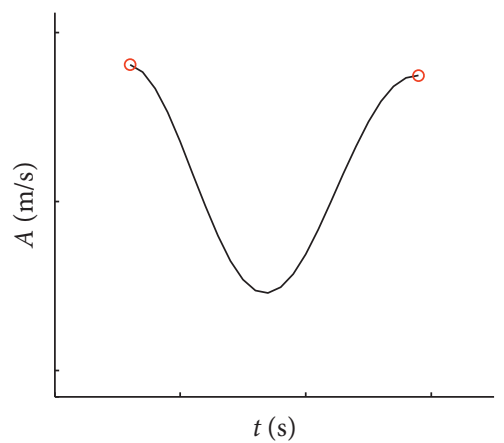

Figure 2: The $\mathrm{V}$ wave.

$$
\begin{aligned}
& m(t)=\frac{1}{n_{k}} \sum_{i=1}^{n_{k}} x\left(t_{i}\right), \quad t_{k} \leq t<t_{k+1}, \\
& a(t)=\frac{x\left(t_{k}\right)+x\left(t_{k+1}\right)}{2}, \quad t_{k} \leq t<t_{k+1},
\end{aligned}
$$

where $n_{k}$ is the number of samples contained between the $k$ th maximum and the $k+1$ th maximum.

According to formula (2), the local frequency is defined as the reciprocal of the local period, i.e.,

$$
v(t)=\frac{1}{T(t)}=\frac{1}{t_{k+1}-t_{k}}, \quad t_{k} \leq t<t_{k+1},
$$

where the local frequency $v(t)$ represents the number of times completing the vibration in a unit of local time. It is used to measure the speed of local vibration, and the unit is still $\mathrm{Hz}$.

The characteristic curves of local amplitude $a(t)$, local mean $m(t)$ and local frequency $v(t)$ calculated by equations (3) to (4) are all broken lines. In order to improve the accuracy of analysis results and to get a smoother curve, the moving average (MA) technology is applied for processing the curves, and the principle is as follows [39].

Let the original broken line sequence be $\{y(i) \mid i=$ $1,2,3, \ldots, n\}$. Every point after being smoothed should satisfy the formula as follows:

$$
\begin{aligned}
y^{\prime}(i)= & \frac{1}{2 N+1}[y(i-N)+y(i-N+1)+\ldots+y(i+N-1) \\
& +y(i+N)],
\end{aligned}
$$

where $2 N+1$ is a smooth interval span, $2 N+1<n$.

Obviously, the difference in span selection has a direct influence on the smoothing effect, which will cause different errors in the calculation results of the local feature quantities. For the impact signal, in order to ensure that the impact extreme value is not lost during the smoothing process, it is necessary to adjust the span to $1 / 5$ of the maximum interval of the adjacent maximum value as the best effect. The smoothing result of the characteristic curve of the arbitrary signal $x(t)$ is shown in Figures 3 and 4 .

2.2. Construction of Time-Frequency Distribution Based on Local Frequency. In order to analyze the nonstationary signal, it is necessary to construct a method of time-frequency distribution based on local frequency. According to the local amplitude curve $a(t)$ defined in equation (4), it represents the absolute amplitude of the $\mathrm{V}$-wave in a complete local period. In order to eliminate the influence of the equilibrium position point fluctuation, the local mean curve $m(t)$ should be removed, i.e.,

$$
a^{\prime}(t)=|a(t)-m(t)|,
$$

where $a^{\prime}(t)$ represents the fluctuation amplitude of the local frequency component $v(t)$ respected to the equilibrium position with time.

Plotting the time $t$, the local frequency $v(t)$, and the local amplitude $a^{\prime}(t)$ on a three-dimensional map, and the local amplitude $a^{\prime}(t)$ is represented by a contour of a different color. Then, a typical time-frequency distribution diagram of the signal $x(t)$ based on the local frequency is formed. Figure 5 shows the time-frequency distribution of the signal $x(t)$ is consistent with the local frequency feature extracted in Figure 4.

2.3. Adaptive Waveform Decomposition Algorithm. According to definition of local frequency and its timefrequency distribution, we can only analyze the signal with single component. For many test signals of machine, it usually contains various components from different excitations. So, if we want to extract the time-frequency feature of each component, the multicomponent signal should be decomposed firstly. In the calculation of the traditional frequency and instantaneous frequency of the multicomponent nonstationary signal, the signal can be finally reduced to the following two forms:

$$
\begin{aligned}
& x(t)=\sum_{k=1}^{\infty} c_{k} \sin \left(k \omega t+\theta_{k}\right), \\
& x(t)=\sum_{k=1}^{N} a_{k}(t) \cos \phi_{k}(t)+R(t),
\end{aligned}
$$

where $R(t)$ is the residual term.

The two forms of decomposition have great similarities, all of which express frequency information in a superposition of multiple harmonic waveforms. However, the actual complex signals often do not have harmonic waveform characteristics and may be composed of a limited 


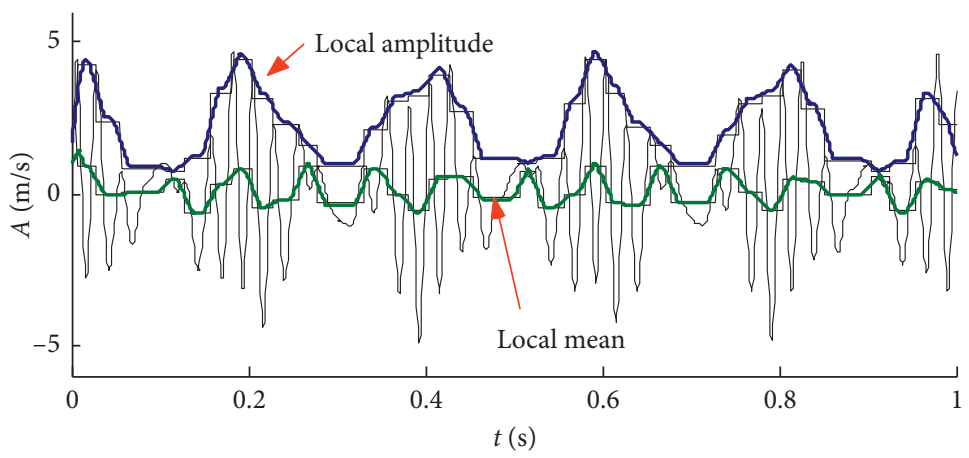

Figure 3: The local amplitude and local mean curve of the signal $x(t)$.

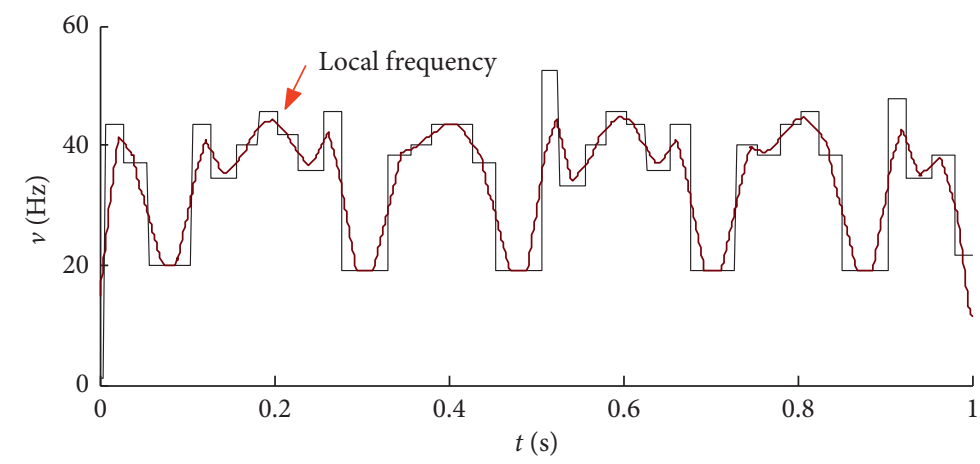

FIgURE 4: The local frequency curve of the signal $x(t)$.

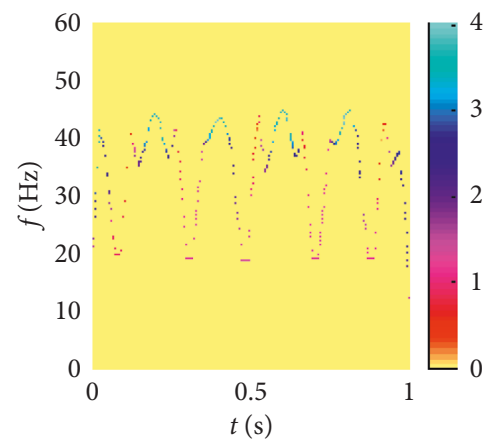

Figure 5: The time-frequency distribution of the signal $x(t)$ based on local frequency.

number of other shape waveforms. Considering the efficiency, accuracy, and frequency physical meaning of signal decomposition, the harmonic waveform combination does not conform to the essential features of the signal. Therefore, based on the local frequency concept, a new adaptive waveform decomposition (AWD) method is proposed to adapt to the feature extraction of nonstationary signal with multicomponents:

$$
x(t)=\sum_{m=1}^{N} s_{m}(t)+W(t),
$$

where $s_{m}(t)$ represents the $m$ th waveform function contained in the original signal and $W(t)$ is the residual term. The implementation process is as follows:
(1) Assume that the discrete time series corresponding to the signal $x(t)$ is $\{x(i) \mid i=1,2,3, \ldots, n\}$. Then, according to formula (12), find the local maxima $n_{1}$ in the signal $x(t)$ as the first layer extreme value sequence $\left\{p_{1}(k) \mid k=1,2,3, \ldots, N_{1}\right\}$.

(2) Calculate the local amplitude $a_{1}(t)$, the local mean $m_{1}(t)$, and the local frequency $v_{1}(t)$ corresponding to the first-order extreme value sequence $p_{1}(t)$ according to formulas (3) to (5).

(3) Smooth $a_{1}(t), m_{1}(t)$, and $v_{1}(t)$ using the moving average technique.

(4) De-average the local amplitude to obtain the amplitude envelope curve $a_{1}^{\prime}(t)$, which is

$$
a_{1}^{\prime}(t)=a(t)-m(t) .
$$

(5) Similarly, find the $n_{2}$ local maxima as the second layer $\left\{p_{2}(k) \mid k=1,2,3, \ldots, N_{2}\right\}$ in the first layer of extreme sequence $\left\{p_{1}(k) \mid k=1,2,3, \ldots, N_{2}\right\}$, continue smoothing and de-averaging processing to obtain the amplitude envelope curve $a_{2}^{\prime}(t)$ according to steps (2), (3), and (4).

(6) Repeat the above steps until you find the m-th order extremum sequence $\left\{p_{m}(k) \mid k=1,2,3, \ldots, N_{m}\right\}$. When it satisfies $N_{m} \leq 2$, the decomposition is ended.

According to the above method, $x(t)$ can obtain $m-1$ amplitude envelope curves $a_{1}^{\prime}(t), a_{2}^{\prime}(t), \ldots, a_{m-1}^{\prime}(t)$, so each component decomposed from $x(t)$ is obtained as follows: 


$$
\left\{\begin{array}{l}
s_{m}(t)=a_{m-1}^{\prime}(t) \\
s_{m-1}(t)=a_{m-2}^{\prime}(t)-a_{m-1}^{\prime}(t) \\
\vdots \\
s_{1}(t)=x-a_{1}^{\prime}(t)
\end{array}\right.
$$

The abovementioned decomposition process only depends on characteristics of the signal $x(t)$ itself, and the local frequency of each components $s_{1}(t), s_{2}(t), \ldots, s_{m}(t)$ does not depend on the harmonic form. Therefore, the proposed AWD method has good adaptability.

2.4. Applicability Analysis of the Proposed Method. In this section, some simulation examples are taken as the research object, and the main characteristics of the proposed method above in time-frequency analysis of multicomponent signals are described in detail by comparing with the EMD (empirical mode decomposition) method [40].

\subsubsection{Combination of Multiple Harmonic Signals}

$$
x_{1}(t)=\sin (2 \pi \cdot 10 \cdot t)+\sin (2 \pi \cdot 30 \cdot t)+\sin (2 \pi \cdot 80 \cdot t) .
$$

Figure 6 shows the decomposition effects of the simulation signal $x_{2}(t)$ based on AWD and EMD. Obviously, both of them can effectively extract the three harmonic waveforms contained in the original signal, which are decomposed from high frequency to low frequency, respectively. As the number of decomposition layers increases, the distance between the first extreme point of the layer and the first data point increases. Whether it is the sliding average method or the spline envelope method, it is difficult to give a satisfactory fit. Although many scholars have given various endpoint extension methods, it has always been ineffective for nonlinear and nonstationary signals.

Figure 7 shows the time-frequency distribution result of the signal $x_{1}(t)$ based on the fusion method of local frequency and AWD, and Figure 8 shows the result based on the fusion method of instantaneous frequency and EMD. It can be seen that both the local frequency and instantaneous frequency can accurately represent the stationary distribution of the three harmonic components in the original signal $x_{1}(t)$. But the time-frequency resolution of the fusion method of local frequency and AWD is higher than the fusion method of instantaneous frequency and EMD.

\subsubsection{Combination of Harmonic Signal and FM-AM Signal.}

$$
\begin{aligned}
x_{2}(t)= & \sin (2 \pi \cdot 30 t)+[1+0.5 \sin (2 \pi \cdot 10 \cdot t)] \\
& \cdot \cos [2 \pi \cdot 150 \cdot t+2 \cos (2 \pi \cdot 5 \cdot t)] .
\end{aligned}
$$

Figure 9 shows the decomposition effects of the simulation signal $x_{2}(t)$ based on AWD and EMD. Similarly, there is still a high degree of coincidence, and the signal $x_{2}(t)$ is adaptively decomposed into three components. It can be seen that the first layer of AWD and EMD result is

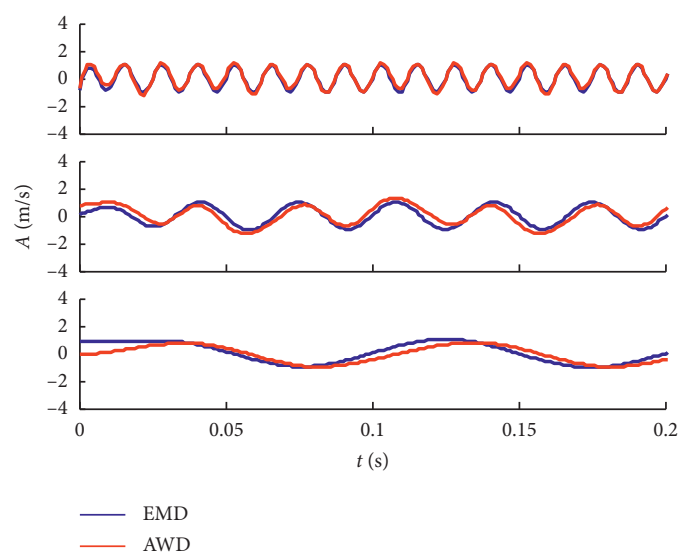

FIGURE 6: The decomposition result of the signal $x_{1}(t)$ based on AWD and EMD.

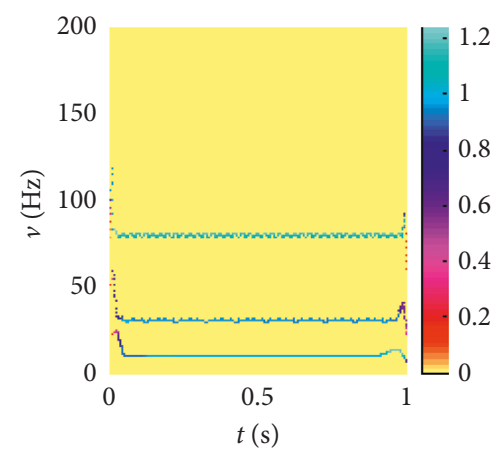

FIGURE 7: The time-frequency distribution of the signal $x_{1}(t)$ based on the local frequency and AWD.

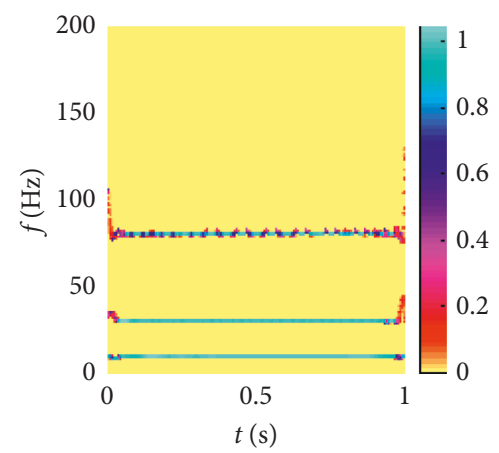

Figure 8: The time-frequency distribution of the signal $x_{1}(t)$ based on the instantaneous frequency and EMD.

the FM-AM component, the second layer is the harmonic component, and the third layer is the trend term.

Figure 10 shows the time-frequency distribution result of the signal $x_{2}(t)$ based on the fusion method of local frequency and AWD, and Figure 11 shows the result based on the fusion method of instantaneous frequency and EMD.

In the time-frequency distribution result of the signal $x_{2}(t)$ based on the fusion method of local frequency and AWD as shown in Figure 10, the $30 \mathrm{~Hz}$ stationary harmonic and FM-AM with $150 \mathrm{~Hz}$ carrier frequency are clearly 


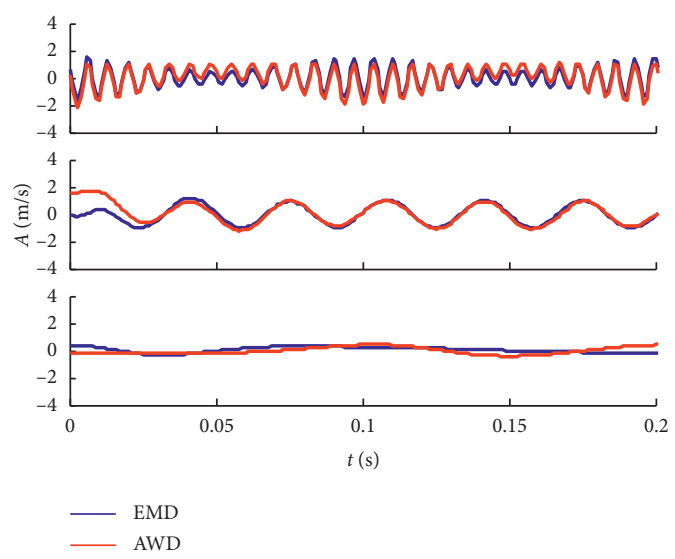

FIgURE 9: The decomposition result of the signal $x_{2}(t)$ based on AWD and EMD.

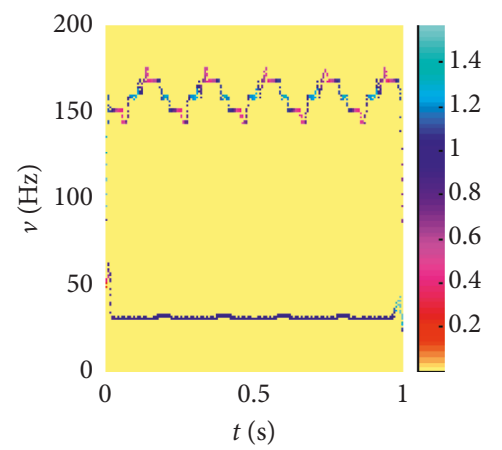

FIGURE 10: The time-frequency distribution of the signal $x_{2}(t)$ based on the local frequency and AWD.

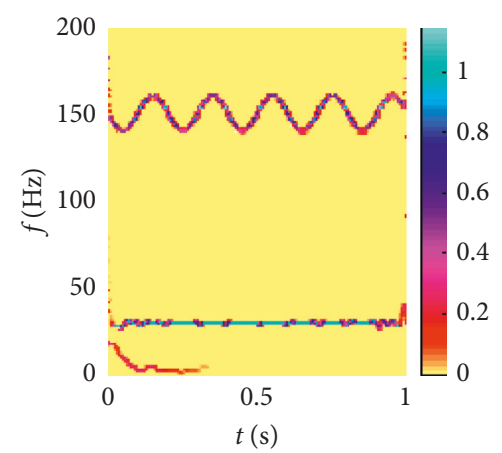

FIGURE 11: The time-frequency distribution of the signal $x_{2}(t)$ based on the instantaneous frequency and EMD.

extracted. However, in Figure 11, the fusion method of instantaneous frequency and EMD generate some interference information at the low frequency because of the trend term. Additionally, comparing Figure 10 with Figure 11, it can be seen that the time-frequency features extracted by using the proposed method in this paper have no false redundant frequency and the resolution is significantly higher.

In summary, by comparing the time-frequency analysis of the multicomponent signals, it is shown that both the
AWD and the EMD can achieve a good adaptive decomposition effect, and both of them have the same accuracy. However, in time-frequency analysis, the local frequency is clearer in resolution than instantaneous frequency, and it not only has clear physical meaning but also contains less interference components. Therefore, the proposed method can accurately obtain the essential features of the frequency components contained in the original signal, and its validity and applicability is verified according to simulation signals above.

\section{Quantitative Measure of the Complexity of Nonlinear Time Series}

3.1. Algorithm of LZC. The definition of complex measure is proposed by Kolmogorov and is characterized by the number of bits of the shortest program required to produce a sequence of symbols. Later, Lempel and Ziv proposed an algorithm to achieve this complexity, called Lempel-Ziv complexity (LZC), which is widely used in nonlinear scientific research [41]. LZC analysis is based on coarse graining of the measurements. Before the LZC measure is calculated, the time series must be transformed into a finite symbol sequence. Generally, an arbitrary time series $\{x(i) \mid i=1,2,3, \cdots, n\}$ is converted into a binary series. By comparison with the threshold, $x(i)$ is converted into a $0-1$ series $\{S(i) \mid i=1,2,3, \cdots, n\}$ as follows [42]:

$$
S(i)= \begin{cases}0, & x(i)<x_{\text {ave }}, \\ 1, & x(i) \geq x_{\text {ave }},\end{cases}
$$

where $x_{\mathrm{ave}}$ is the mean of time series of $x(i)$. Then, the LZC measure can be estimated by using the following algorithm as shown in Figure 12.

The abovementioned algorithm is repeated until $Q$ is the last character. The measure of complexity is $c(n)$. In order to obtain a complexity measure which is independent of the series length, $c(n)$ must be normalized. If the length of the sequence is $n$ and the number of different symbols in the symbol set is 2 , it has been proved that the upper bound of $c(n)$ is given by

$$
\lim _{n \rightarrow \infty} c(n)=b(n)=\frac{n}{\log _{2}(n)},
$$

and $c(n)$ can be normalized via $b(n)$

$$
0 \leq C=\frac{c(n)}{b(n)} \leq 1,
$$

where the normalized complexity index $C$ is called the Lempel-Ziv complexity.

Obviously, equation (17) can be established only if the sample length $n$ is large enough. The literature has given the experience valve $n \geq 3600$ [43]. The complexity index $C$ presents the random degree of time series. For the completely random time series, $C$ is close to 1 . On the contrary, the periodic time series is close to 0 . In practice, the vibration signals of reciprocating compressor vary with each fault states randomly, so the LZC can be extracted as a useful measure for machine health condition feature. 


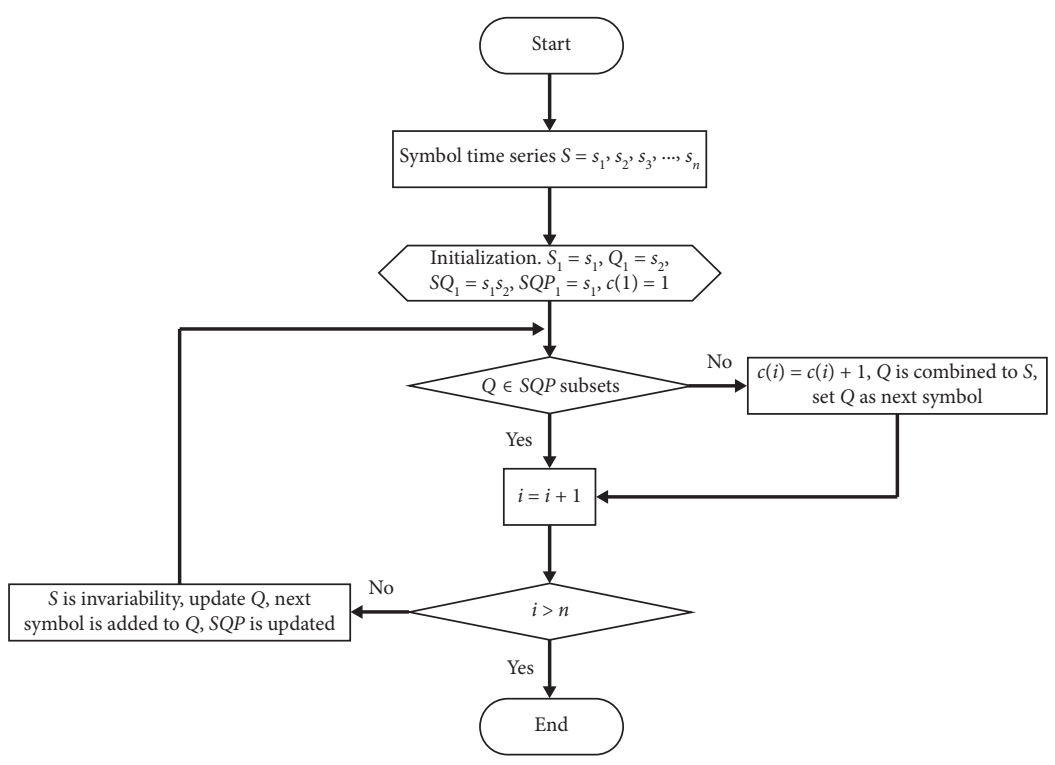

FIgURE 12: The principle diagram of LZC.

3.2. Performance Experiment of LZC. To identify the effectiveness of LZC method, the typical nonlinear dynamical system is taken as an example to investigate the performance of LZC. Consider the following logistic map [44]

$$
x_{n+1}=\lambda \cdot x_{n} \cdot\left(1-x_{n}\right) \text {, }
$$

where $x_{\mathrm{n}} \in[0,1], \lambda \in[2.8,4], \lambda$ is the control parameters, and the step size is 0.01 . The sample length of nonlinear time series is $N=5000$.

According to the LZC algorithm, the complexity index $C$ of logistic map with different parameters $\lambda$ can be calculated. The result is shown in Figure 13.

Observe Figures 13(a) and 13(b), it indicates the dynamical behaviour of logistic map with different control parameters $\lambda$. The trends of Lyapunov exponent and the complexity index $C$ are much synchronous. When $2.8 \leq \lambda<3.57$, the Lyapunov exponent is less than zero and it shows that the logistic system enters in the periodic or quasiperiodic region. Moreover, most values of LZC are close to zero. When $3.57<\lambda \leq 4$, Lyapunov exponent is greater than zero, it indicates that the system enters the chaos region. Meanwhile, the complexity index $C$ tends to be around 0.5. During this region, the Lyapunov exponent less than zero including $\lambda=3.63, \lambda=3.74$, and $\lambda=3.83$ are called periodic windows. In these regions, the complexity index $C$ is still close to zero.

In summary, LZC can be used as identifying the dynamical behaviour of nonlinear system effectively. The analysis results are stable and reliable when the number of samples increase. Therefore, the LZC value of different system states can be compared together without any prior knowledge about fault mechanism.

\section{Application in Fault Feature Extraction of Rolling Bearings}

4.1. Preprocessing Analysis of Vibration Signal. To reduce the influence of noise on the nonlinear and nonstationary measure analysis results of multisource impact signals of gas valve, the preprocessing based on AWD and mutual information (MI) fusion noise reduction technology was proposed. The definition of MI can be given as follows:

$$
\begin{aligned}
I(X, Y) & =H(X)+H(Y)-H(X, Y) \\
& =\sum_{i=1}^{n} \sum_{j-1}^{m} P\left(x_{i}, y_{j}\right) \log \frac{p\left(x_{i}, y_{j}\right)}{p\left(x_{i}\right) p\left(y_{j}\right)},
\end{aligned}
$$

where $p(x), p(y)$, and $p(x, y)$ denote the probability density function of two random variables $X, Y$ and the joint probability distribution of $(X, Y)$. MI measures the correlation degree of two signal. When they are absolute, MI value will be 0 . When they are fully the same, the value will be 1 .

According to the proposed AWD method in this paper, the original vibration signal with background noise can be adaptively decomposed into a series of waveform component signals $s_{1}(t), s_{2}(t), \ldots, s_{m}(t)$. The MI value of each component signal and the original signal were calculated following the steps of equation (18), respectively. By sorting, one or more correlated component signals are selected to be recombined to form a noise reduction signal.

4.2. LZC Feature Extraction of Rolling Bearing in Time Domain. In this section, vibration signals of the rolling bearings under different faults are taken as an application object. The standard data are obtained from the bearing data center of the Electrical Engineering Laboratory of Case Western Reserve University, and its experimental bench structure is shown in Figure 14. In the experiment, the deep groove ball bearing was selected at the motor drive end. The detailed parameters are shown in Table 1. In this experiment, the tiny pits of the inner ring, the outer ring, and the rolling element of the bearing are processed by the electric spark machine to simulate the single-point damage fault, diameter, and depth size are $0.1778 \mathrm{~mm} \times 0.2794 \mathrm{~mm}$, 


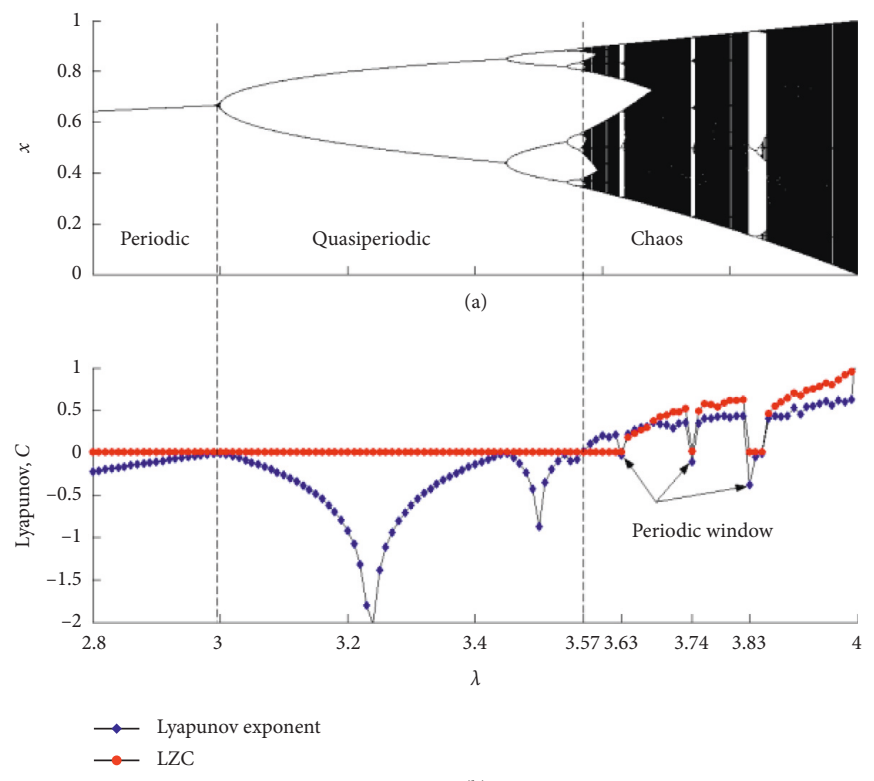

FIgURE 13: The logistic map with different parameters $\lambda$. (a) Bifurcation diagram; (b) comparison of Lyapunov exponent and LZC.

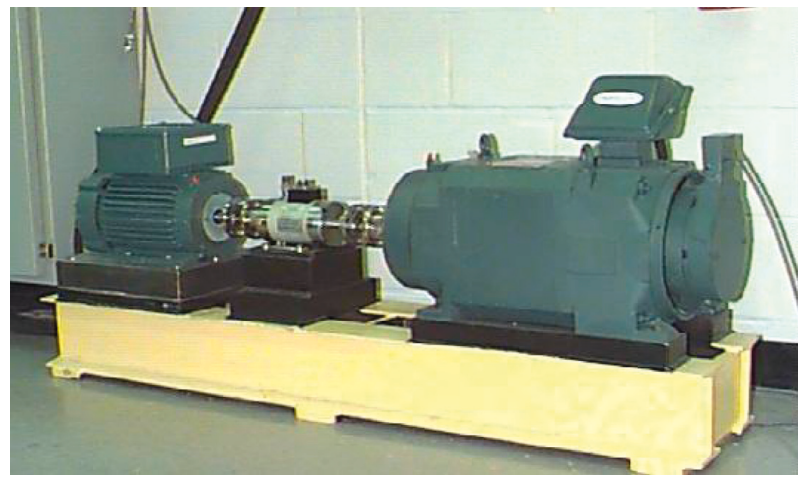

FIGURE 14: The fault simulation experimental bench of rolling bearing.

TABLE 1: Parameters of rolling bear.

\begin{tabular}{lcc}
\hline No. & Parameter (unit) & Value \\
\hline 1 & Number of ball roller $z$ & 9 \\
2 & Diameter of ball roller $d(\mathrm{~mm})$ & 7.94 \\
3 & Diameter of rolling bear $D(\mathrm{~mm})$ & 39 \\
4 & Contact angle $\alpha\left(^{\circ}\right)$ & 90 \\
\hline
\end{tabular}

$0.3556 \mathrm{~mm} \times 0.2794 \mathrm{~mm}, \quad 0.5334 \mathrm{~mm} \times 0.2794 \mathrm{~mm}, \quad$ and $0.7112 \mathrm{~mm} \times 1.27 \mathrm{~mm}$, respectively. The sampling frequency $f_{\mathrm{s}}$ is $12000 \mathrm{~Hz}$, the running speed is set separately into $1797 \mathrm{r} / \mathrm{min}, 1772 \mathrm{r} / \mathrm{min}, 1750 \mathrm{r} / \mathrm{min}$, and $1730 \mathrm{r} / \mathrm{min}$, which are corresponding to fundamental frequency $f_{0}$ of the rolling bearings $29.95 \mathrm{~Hz}, 29.53 \mathrm{~Hz}, 29.17 \mathrm{~Hz}$, and $28.83 \mathrm{~Hz}$. Where the fault position of the outer ring is selected at the 6 o'clock direction, which is the main load position.

According to the parameters in Table 1, taking the speed of $1750 \mathrm{r} / \mathrm{min}$ as an example, the frequency features of faults in each part can be calculated separately as follows:

$$
\begin{aligned}
& f_{\mathrm{IR}}=\frac{1}{2} z\left(1+\frac{d}{D} \cos \alpha\right) f_{0}=157.94 \mathrm{~Hz}, \\
& f_{\mathrm{OR}}=\frac{1}{2} z\left(1-\frac{d}{D} \cos \alpha\right) f_{0}=104.56 \mathrm{~Hz}, \\
& f_{\mathrm{BE}}=\frac{1}{2} \frac{D}{d}\left(1-\left(\frac{d}{D}\right)^{2} \cos ^{2} \alpha\right) f_{0}=137.48 \mathrm{~Hz},
\end{aligned}
$$

where $f_{\mathrm{IR}}, f_{\mathrm{OR}}, f_{\mathrm{BE}}$, and $f_{0}$ are corresponding to the fault frequency of the inner ring, the outer ring, the rolling element, and the fundamental frequency of rotation speed, respectively.

Figure 15 shows the time domain waveform of the vibration signal of rolling bearing in four states, including the normal state, inner ring fault state, outer ring fault state, and rolling element fault state. The rotation speed is $1750 \mathrm{r} / \mathrm{min}$ and the damage size is $0.5334 \mathrm{~mm} \times 0.2794 \mathrm{~mm}$. It can be seen that the vibration signals in the fault state of the inner ring and the outer ring have obvious impact feature, while the features of the rolling element fault state and the normal state are not obvious. It shows that the vibration amplitude of the normal state is the smallest and the the amplitude of the outer ring state is the largest. This is mainly because the selected outer ring fault position is in the main bearing direction, and the load of the rotor increases the impact energy of the rolling element falling into the outer ring.

Figure 16 shows the AWD results of the above four states of rolling bearings. The vibration signals in each state are adaptively decomposed into six components. As the number of decomposition layers increases, the frequency gradually changes from the high-frequency band to the low-frequency band. The components of each layer in the normal state do not have obvious impact features, the signal-to-noise ratio is 


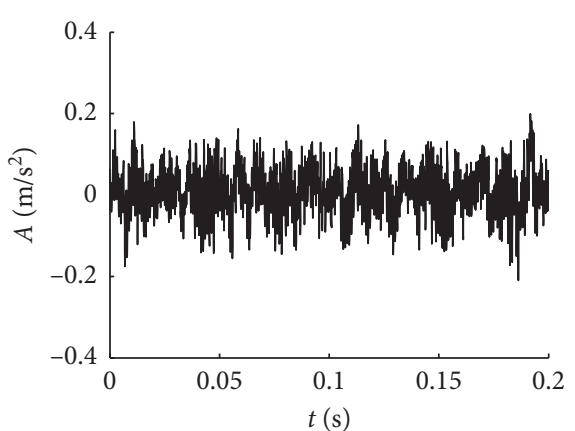

(a)

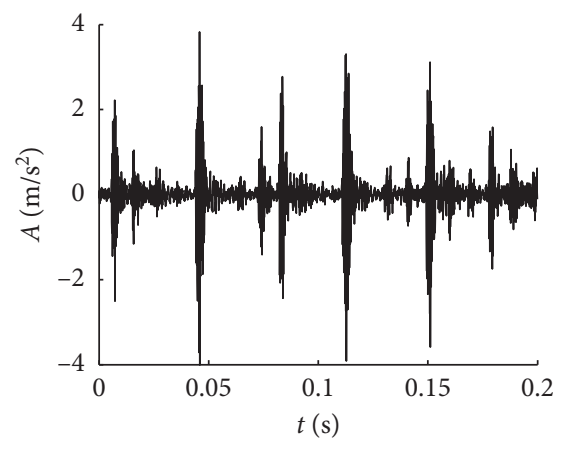

(c)

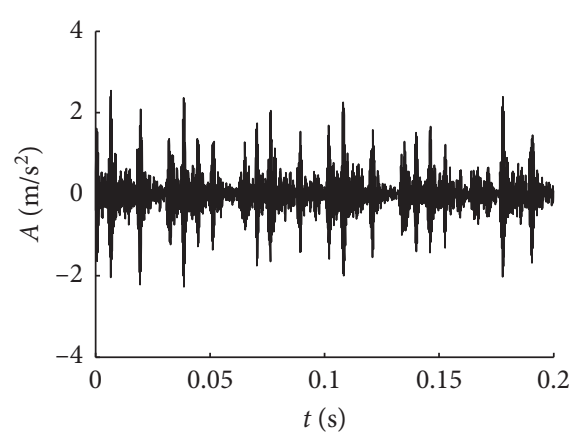

(b)

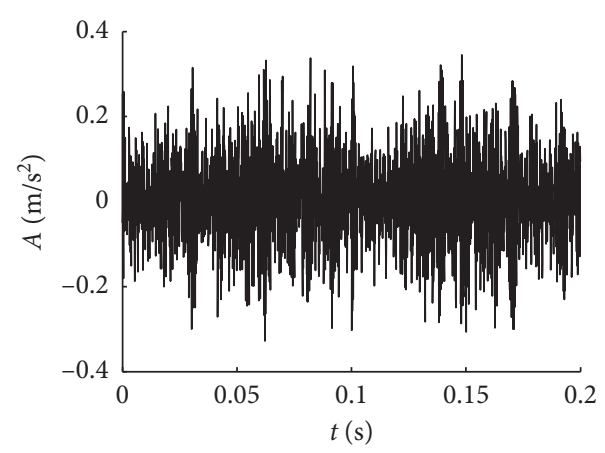

(d)

FIGURE 15: The time domain waveform in four state of rolling bearings: (a) normal state, (b) inner ring fault state, (c) outer ring fault state, and (d) rolling element fault state.

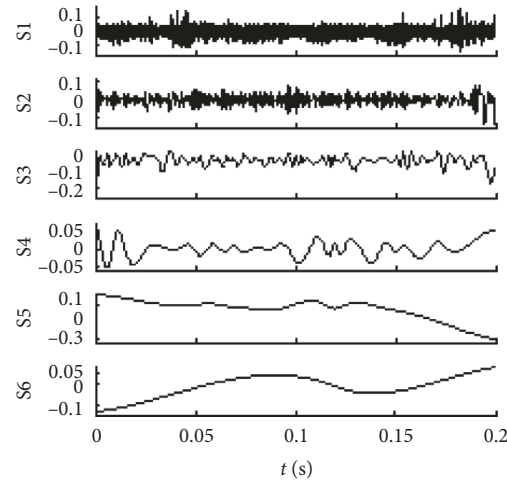

(a)

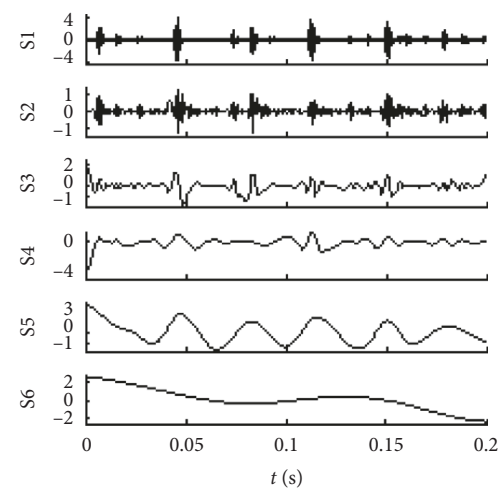

(c)

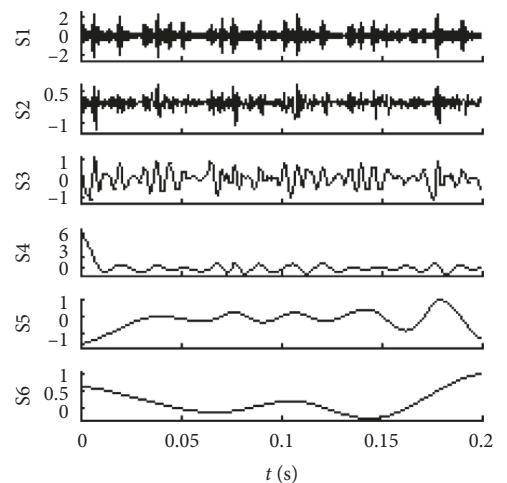

(b)

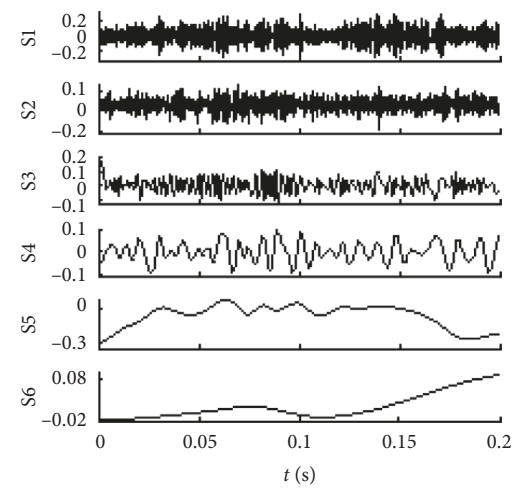

(d)

FIgURE 16: The AWD results in four state of rolling bearings: (a) normal state, (b) inner ring fault state, (c) outer ring fault state, and (d) rolling element fault state. 
relatively low, and the main feature of components is not easily recognized. The first three components of the inner ring fault state present the impact features, and the latter three components present the harmonic features. However, the outer ring fault state and the rolling element fault state have the significant periodic impact features in the first four components, and the remaining component are low-frequency harmonic periodic features. In addition, the noise content of the first and second layer waveform components in each state is larger than that of the other layer components, and the energy of the signal is mainly concentrated in the first two components.

According to the analysis of AWD results, we can easily obtain the main components and then the Lempel-Ziv complex measure analysis is performed on each signal by using the LZC algorithm. Table 2 shows the LZC features of the timedomain signal for the rolling bearing in four states at different speeds with the damage size $0.5334 \mathrm{~mm} \times 0.2794 \mathrm{~mm}$. Table 3 shows the LZC features of the time-domain signal for the rolling bearings in fault states under different damage diameters with the speed $1750 \mathrm{r} / \mathrm{min}$.

It can be seen from Table 2 that the LZC values of rolling bear in the inner ring fault state are gradually decreased with the speed decreased from $1797 \mathrm{r} / \mathrm{min}$ to $1730 \mathrm{r} / \mathrm{min}$, but the LZC values are increased in the other three states. The reason may be as follows. With the decreasing of the rotational, the bearing load increases relatively, and the modulation effect on the signal is more significant. The contact between the outer ring and the rolling element is basically fixed, and its main role is frequency modulation. So the new frequency change is increased. The complexity of the signal leads to a higher LZC value. However, when the inner ring rotates with the axis, the contact area of pitting fault changes continuously, and its main role is to generate a low-frequency amplitude modulation. It is different from frequency modulation that the signal is affected globally in the lowfrequency oscillation mode. Therefore, the periodicity is enhanced and the randomness is reduced, which results in a lower LZC value.

In Table 3, with the increasing of the damage diameter from $0.1778 \mathrm{~mm}$ to $0.7112 \mathrm{~mm}$, the LZC values of rolling bear in the inner ring fault state are gradually decreased, while the LZC values are decreased in the other three states. That is because the rotation speed is constant, the load is relatively stable, the damage area is enlarged, the actual contact area between the rolling element and the outer ring is reduced, the contact pressure is rapidly increased and decreased, and the impact feature is more obvious. The influence is dominant, reflecting a wider frequency band and harmonics on the spectrum. The result indicates that the randomness of the original signal is more prominent and it results in a higher LZC value. However, due to the influence of the low frequency, the vibration signal of inner ring presents the amplitude modulation feature. The order of original signal is enhanced and the randomness is reduced, which results in a lower LZC value. Domain. According to the analysis above, we can find that
TABLE 2: The LZC features of the time-domain signal for the rolling bearing at different speeds.

\begin{tabular}{lcccc}
\hline $\begin{array}{l}\text { Rotation speed } \\
\text { (r/min) }\end{array}$ & Normal & $\begin{array}{c}\text { Inner ring } \\
\text { fault }\end{array}$ & $\begin{array}{c}\text { Outer ring } \\
\text { fault }\end{array}$ & $\begin{array}{c}\text { Rolling } \\
\text { element fault }\end{array}$ \\
\hline 1797 & 0.1503 & 0.2089 & 0.0968 & 0.2879 \\
1772 & 0.1962 & 0.2013 & 0.1655 & 0.2953 \\
1750 & 0.2017 & 0.1936 & 0.2885 & 0.3032 \\
1730 & 0.2115 & 0.1860 & 0.3614 & 0.3236 \\
\hline
\end{tabular}

TABLE 3: The LZC features of the time-domain signal for the rolling bearing under different damage diameters.

\begin{tabular}{lccc}
\hline $\begin{array}{l}\text { Damage size } \\
(\mathrm{mm})\end{array}$ & $\begin{array}{c}\text { Inner ring } \\
\text { fault }\end{array}$ & $\begin{array}{c}\text { Outer ring } \\
\text { fault }\end{array}$ & $\begin{array}{c}\text { Rolling element } \\
\text { fault }\end{array}$ \\
\hline 0.1778 & 0.2726 & 0.2038 & 0.2777 \\
0.3556 & 0.2573 & 0.2404 & 0.2955 \\
0.5334 & 0.1936 & 0.2885 & 0.3032 \\
0.7112 & 0.1420 & 0.3013 & 0.3140 \\
\hline
\end{tabular}

the time domain features of vibration signal the rolling bearing as shown in Figure 15 cannot directly and accurately identify the characteristic frequency, especially for distinguishing the normal state and the fault state of the rolling element with low signal-to-noise ratio. For the complex nonstationary signal of rolling bearing, in this section, the time-frequency analysis based on the local frequency and AWD method is applied. The result is shown in Figure 17.

In Figure 17, the time-frequency distribution diagram of the vibration signal in four states of the rolling bearing shows a typical AM-FM features, and the local frequency fluctuates around a certain constant value with time. The carrier frequency in the normal state is $88 \mathrm{~Hz}$, which is near the triple frequency of the fundamental frequency, and the carrier frequencies in the inner ring fault state, the outer ring fault state, and the rolling element fault state are $158 \mathrm{~Hz}$, $105 \mathrm{~Hz}$, and $138 \mathrm{~Hz}$, respectively. This is consistent with the theoretical frequency of each fault state calculated by equations (18)-(20), which can further verify the effectiveness of the proposed method. In addition, according to the time-frequency analysis, it is obvious that the feature of the rolling element fault is the most ambiguous, the fluctuation is the most severe, and the noise is the most serious. However, the time-frequency distribution of the vibration signal under normal condition is relatively stable.

In order to accurately quantify these time-frequency feature of rolling bearings, the Lempel-Ziv complexity analysis method is applied. Table 4 shows the LZC comparison results of the rolling bearing in the time-frequency domain at different speeds, and Table 5 shows the LZC comparison results of the rolling bearing in time-frequency domain under different damage diameters. It can be seen that the structural complexity of time-frequency distribution in each state of the rolling bearing has a similar change law with the time domain. With the decreasing of the rotational speed or the improvement of the damage degree, the LZC values of time-frequency distribution in the inner ring fault gradually decrease but they are increased in the other three states. The result indicates that the time-frequency transform 


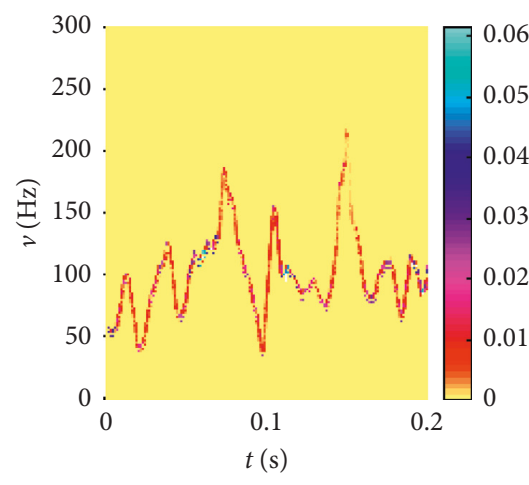

(a)

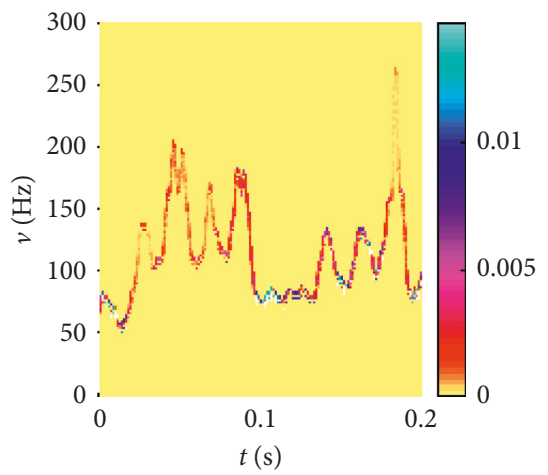

(c)

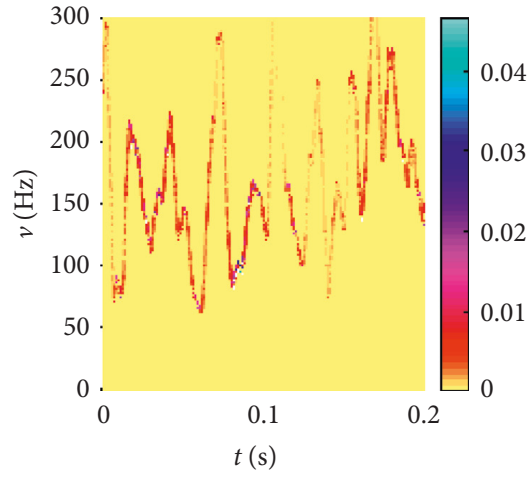

(b)

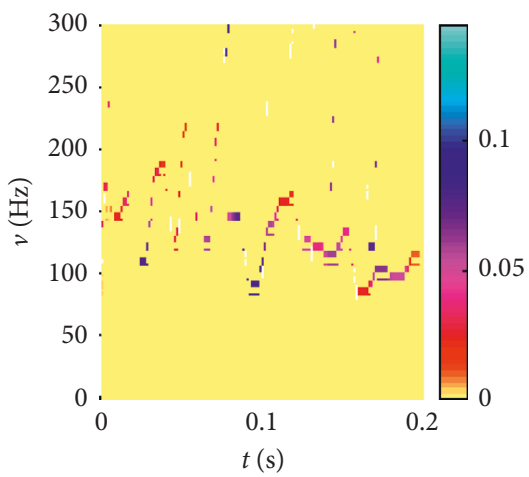

(d)

FIGURE 17: The time-frequency distribution in four state of rolling bearings: (a) normal state, (b) inner ring fault state, (c) outer ring fault state, and (d) rolling element fault state.

TABLE 4: The LZC features of time-frequency distribution for the rolling bearing at different speeds.

\begin{tabular}{lcccc}
\hline Rotation speed $(\mathrm{r} / \mathrm{min})$ & Normal & Inner ring fault & Outer ring fault & Rolling element fault \\
\hline 1797 & 0.0459 & 0.0637 & 0.0764 & 0.0841 \\
1772 & 0.0507 & 0.0588 & 0.0825 & 0.0912 \\
1750 & 0.0611 & 0.0433 & 0.1188 & 0.1121 \\
1730 & 0.0688 & 0.0312 & 0.1217 & 0.1172 \\
\hline
\end{tabular}

TABLE 5: The LZC features of time-frequency distribution for the rolling bearing under different damage diameters.

\begin{tabular}{lccc}
\hline Damage size $(\mathrm{mm})$ & Inner ring fault & Outer ring fault & Rolling element fault \\
\hline 0.1778 & 0.0611 & 0.0866 & 0.0815 \\
0.3556 & 0.0543 & 0.0968 & 0.0923 \\
0.5334 & 0.0433 & 0.1188 & 0.1121 \\
0.7112 & 0.0341 & 0.1424 & 0.1510 \\
\hline
\end{tabular}

preserves the nature of randomness and nonlinearity of the original signal itself, which can easily and quickly quantify the complexity of different fault states. In addition, compared with the LZC value in the time domain, the LZC value in the time-frequency domain is correspondingly reduced. Therefore, the time-frequency analysis can reduce the structural complexity of the signal, making the fault feature clearer and clearer and suppressing the randomness of the noise to some extent. The role of sexual interference has laid a good foundation for the fault diagnosis of rolling bearings.

\section{Conclusions}

A novel local frequency concept and AWD algorithm is proposed for the purpose of time-frequency feature extraction for the vibration signal of rolling bearings presented multisource impact, nonlinear, and nonstationary characteristics. Compared with the commonly used method of time-frequency analysis based on traditional frequency or instantaneous frequency, the proposed method develops the limitation of frequency definition on 
nonharmonic signal, and the physical meaning is clearer. Additionally, the time-frequency features extracted by the AWD and local frequency method have good adaptability, the decomposition result is completely independent, and the frequency band is high to low. It can avoid the false or cross component caused by the forced decomposition of the traditional Fourier transform in the form of harmonic basis function. Based on the proposed method in this study, the LZC algorithm is appled to quantitatively measure the complexity of time-frequency features for vibration signals of the rolling bearings. Therefore, it can be used as an effective method for fault diagnosis of rolling bearings.

\section{Data Availability}

The data used to support the findings of this study are included within the article.

\section{Conflicts of Interest}

The authors declare that there are no conflicts of interest regarding the publication of this paper.

\section{Acknowledgments}

This work was supported by the Natural Science Foundation of China (51505079) and University Nursing Program for Young Scholars with Creative Talents in Heilongjiang Province (UNPYSCT-2015078).

\section{References}

[1] Y. Qin, "A new family of model-based impulsive wavelets and their sparse representation for rolling bearing fault diagnosis," IEEE Transactions on Industrial Electronics, vol. 65, no. 3, pp. 2716-2726, 2018.

[2] H. Shao, H. Jiang, Y. Lin, and X. Li, "A novel method for intelligent fault diagnosis of rolling bearings using ensemble deep auto-encoders," Mechanical Systems and Signal Processing, vol. 102, pp. 278-297, 2018.

[3] A. Glowacz, W. Glowacz, Z. Glowacz, and J. Kozik, "Early fault diagnosis of bearing and stator faults of the single-phase induction motor using acoustic signals," Measurement, vol. 113, pp. 1-9, 2018.

[4] S. Xiao, S. Liu, F. Jiang, M. Song, and S. Cheng, "Nonlinear dynamic response of reciprocating compressor system with rub-impact fault caused by subsidence," Journal of Vibration and Control, vol. 25, no. 11, pp. 1737-1751, 2019.

[5] M. Cerrada, R.-V. Sánchez, C. Li et al., "A review on datadriven fault severity assessment in rolling bearings," $\mathrm{Me}$ chanical Systems and Signal Processing, vol. 99, pp. 169-196, 2018.

[6] H. D. Shao, H. K. Jiang, X. Q. Li, and S. P. Wu, "Intelligent fault diagnosis of rolling bearing using deep wavelet autoencoder with extreme learning machine," Knowledge-Based Systems, vol. 140, pp. 1-14, 2018.

[7] L. Wang, Z. Liu, Q. Miao, and X. Zhang, "Complete ensemble local mean decomposition with adaptive noise and its application to fault diagnosis for rolling bearings," Mechanical Systems and Signal Processing, vol. 106, pp. 24-39, 2018.
[8] Q. He, E. Wu, and Y. Pan, "Multi-scale stochastic resonance spectrogram for fault diagnosis of rolling element bearings," Journal of Sound and Vibration, vol. 420, pp. 174184, 2018.

[9] Y. Cheng, N. Zhou, W. Zhang, and Z. Wang, "Application of an improved minimum entropy deconvolution method for railway rolling element bearing fault diagnosis," Journal of Sound and Vibration, vol. 425, pp. 53-69, 2018.

[10] L. Cui, B. Li, J. Ma, and Z. Jin, "Quantitative trend fault diagnosis of a rolling bearing based on Sparsogram and Lempel-Ziv," Measurement, vol. 128, pp. 410-418, 2018.

[11] A. Rai and S. H. Upadhyay, "A review on signal processing techniques utilized in the fault diagnosis of rolling element bearings," Tribology International, vol. 96, pp. 289-306, 2016.

[12] Z. Q. Ma, W. Ruan, M. Chen, and X. Li, “An improved timefrequency analysis method for instantaneous frequency estimation of rolling bearing," Shock and Vibration, vol. 2018, Article ID 8710190, 18 pages, 2018.

[13] M. J. Roberts, Signals and Systems: Analysis Using Transform Methods and MATLAB, McGraw-Hill, New York, NY, USA, 2nd edition, 2011.

[14] D. Vakman, "On the analytic signal, the Teager-Kaiser energy algorithm, and other methods for defining amplitude and frequency," IEEE Transactions on Signal Processing, vol. 44, no. 1, pp. 791-797, 1996.

[15] S. Xu, L. Feng, Y. Chai, and Y. He, "Analysis of A-stationary random signals in the linear canonical transform domain," Signal Processing, vol. 146, pp. 126-132, 2018.

[16] D. Gabor, "Theory of communication. Part 1: the analysis of information," Journal of the Institution of Electrical Engineers-Part III: Radio and Communication Engineering, vol. 93, no. 26, pp. 429-441, 1946.

[17] I. Daubechies, "Orthonormal bases of compactly supported wavelets," Communications on Pure and Applied Mathematics, vol. 41, no. 7, pp. 909-996, 1988.

[18] F. J. Harris, "On the use of windows for harmonic analysis with the discrete Fourier transform," Proceedings of the IEEE, vol. 66, no. 1, pp. 51-83, 1978.

[19] D. Griffin and J. Lim, "Signal estimation from modified shorttime Fourier transform," IEEE Transactions on Acoustics, Speech, and Signal Processing, vol. 32, no. 2, pp. 236-243, 1984.

[20] I. Daubechies, "The wavelet transform, time-frequency localization and signal analysis," IEEE Transactions on Information Theory, vol. 36, no. 5, pp. 961-1005, 1990.

[21] J. Wang, Q. Wei, L. Zhao, T. Yu, and R. Han, "An improved empirical mode decomposition method using second generation wavelets interpolation," Digital Signal Processing, vol. 79, pp. 164-174, 2018.

[22] S. Mouatadid, J. F. Adamowski, M. K. Tiwari, and J. M. Quilty, "Coupling the maximum overlap discrete wavelet transform and long short-term memory networks for irrigation flow forecasting," Agricultural Water Management, vol. 219, pp. 72-85, 2019.

[23] J. Wang, Y. Han, L. M. Wang, P. Z. Zhang, and P. Chen, "Instantaneous frequency estimation for motion echo signal of projectile in bore based on polynomial chirplet transform," Russian Journal of Nondestructive Testing, vol. 54, no. 1, pp. 44-54, 2018.

[24] J. Tan, "Atomic decomposition of variable Hardy spaces via Littlewood-Paley-Stein theory," Annals of Functional Analysis, vol. 9, no. 1, pp. 87-100, 2018.

[25] Z. J. He, Y. Y. Zi, X.-F. Chen, and X.-D. Wang, "Transform principle of inner product for fault diagnosis," Journal of Vibration Engineering, vol. 20, no. 5, pp. 528-533, 2007. 
[26] N. E. Huang, Z. Shen, S. R. Long et al., "The empirical mode decomposition and the Hilbert spectrum for nonlinear and non-stationary time series analysis," Proceedings of the Royal Society of London. Series A: Mathematical, Physical and Engineering Sciences, vol. 454, no. 1971, pp. 903-995, 1998.

[27] J. S. Smith, "The local mean decomposition and its application to EEG perception data," Journal of the Royal Society Interface, vol. 2, no. 5, pp. 443-454, 2005.

[28] J. S. Cheng, J. D. Zheng, and Y. Yang, “A nonstationary signal analysis approach-the local characteristic-scale decomposition method," Journal of Vibration Engineering, vol. 25, no. 2, pp. 215-220, 2012.

[29] Y. Qin, Y. Mao, and B. Tang, "Multicomponent decomposition by wavelet modulus maxima and synchronous detection," Mechanical Systems and Signal Processing, vol. 91, pp. 57-80, 2017.

[30] Y. Qin, J. Zou, B. Tang, Y. Wang, and H. Chen, "Transient feature extraction by the improved orthogonal matching pursuit and K-SVD algorithm with adaptive transient dictionary," IEEE Transactions on Industrial Informatics, 2019.

[31] P. C. Müller, "Calculation of Lyapunov exponents for dynamic systems with discontinuities," Chaos, Solitons \& Fractals, vol. 5, no. 9, pp. 1671-1681, 1995.

[32] D. Logan and J. Mathew, "Using the correlation dimension for vibration fault diagnosis of rolling element bearings-I. Basic concepts," Mechanical Systems and Signal Processing, vol. 10, no. 3, pp. 241-250, 1996.

[33] D. Wu, S. Zhang, H. Zhao, and X. Yang, "A novel fault diagnosis method based on integrating empirical wavelet transform and fuzzy entropy for motor bearing," IEEE Access, vol. 6, pp. 35042-35056, 2018.

[34] Y. Li, G. Li, Y. Yang, X. Liang, and M. Xu, "A fault diagnosis scheme for planetary gearboxes using adaptive multi-scale morphology filter and modified hierarchical permutation entropy," Mechanical Systems and Signal Processing, vol. 105, pp. 319-337, 2018.

[35] L. Liu, D. Li, and F. Bai, "A relative Lempel-Ziv complexity: application to comparing biological sequences," Chemical Physics Letters, vol. 530, pp. 107-112, 2012.

[36] V. Alexeenko, J. A. Fraser, A. Dolgoborodov et al., "The application of Lempel-Ziv and Titchener complexity analysis for equine telemetric electrocardiographic recordings," Scientific Reports, vol. 9, no. 1, p. 2619, 2019.

[37] W. Henkel, G. Muskhelishvili, D. Nigatu, and P. Sobetzko, The DNA from a Coding Perspective, Information-and Communication Theory in Molecular Biology, Springer, Cham, Switzerland, 2018.

[38] K. Yu, J. Tan, and T. Lin, "Fault diagnosis of rolling element bearing using multi-scale Lempel-Ziv complexity and Mahalanobis distance criterion," Journal of Shanghai Jiaotong University (Science), vol. 23, no. 5, pp. 696-701, 2018.

[39] X. K. Chai, X. H. Weng, Z. M. Zhang, Y. T. Lu, G. T. Liu, and H. J. Niu, "Quantitative EEG in mild cognitive impairment and Alzheimer's disease by AR-spectral and multi-scale entropy analysis," in Proceedings of the World Congress on Medical Physics and Biomedical Engineering 2018, Springer, Prague, Czech Republic, June 2018.

[40] L. Angrisani and M. D’Arco, “A measurement method based on a modified version of the chirplet transform for instantaneous frequency estimation," IEEE Transactions on Instrumentation and Measurement, vol. 51, no. 4, pp. 704-711, 2002.
[41] A. Lempel and J. Ziv, "On the complexity of finite sequences," IEEE Transactions on Information Theory, vol. 22, no. 1, pp. 75-81, 1976.

[42] J. Ziv and A. Lempel, "A universal algorithm for sequential data compression," IEEE Transactions on Information Theory, vol. 23, no. 3, pp. 337-343, 1977.

[43] H. Hong and M. Liang, "Fault severity assessment for rolling element bearings using the Lempel-Ziv complexity and continuous wavelet transform," Journal of Sound and Vibration, vol. 320, no. 1-2, pp. 452-468, 2009.

[44] S. C. Phatak and S. S. Rao, "Logistic map: a possible randomnumber generator," Physical Review E, vol. 51, no. 4, pp. 3670-3678, 1995. 


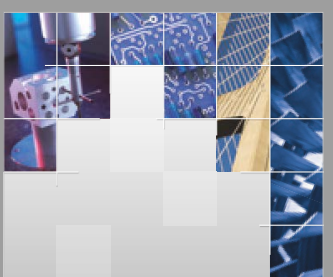

\section{Enfincering}
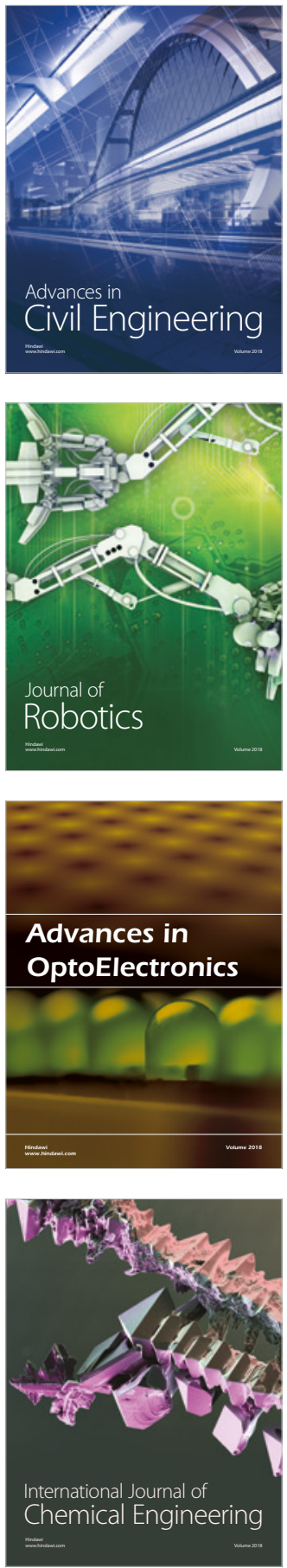

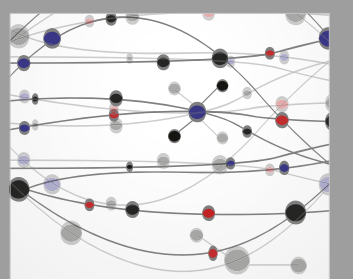

\section{Rotating \\ Machinery}

The Scientific World Journal

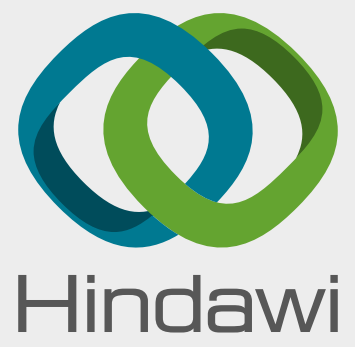

Submit your manuscripts at

www.hindawi.com
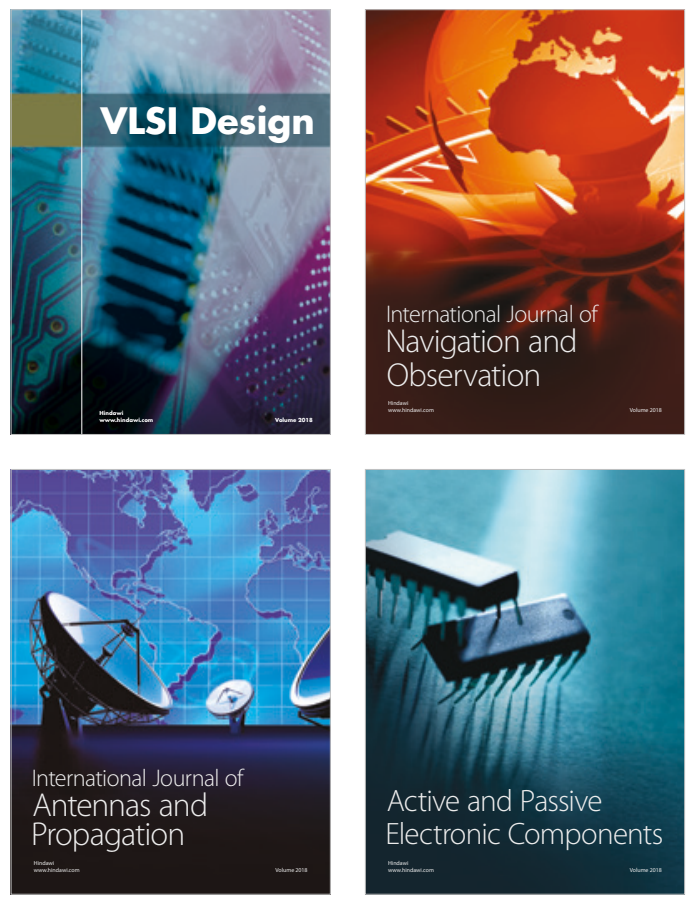
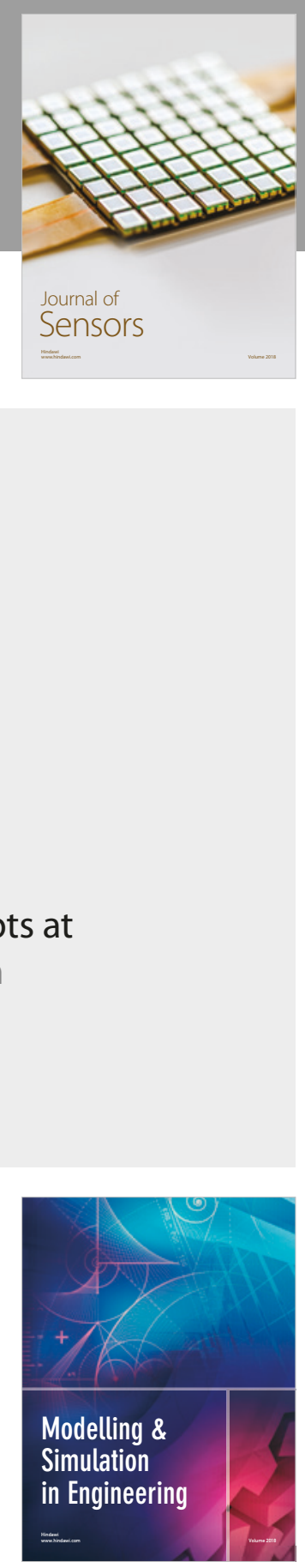

\section{Advances \\ Multimedia}
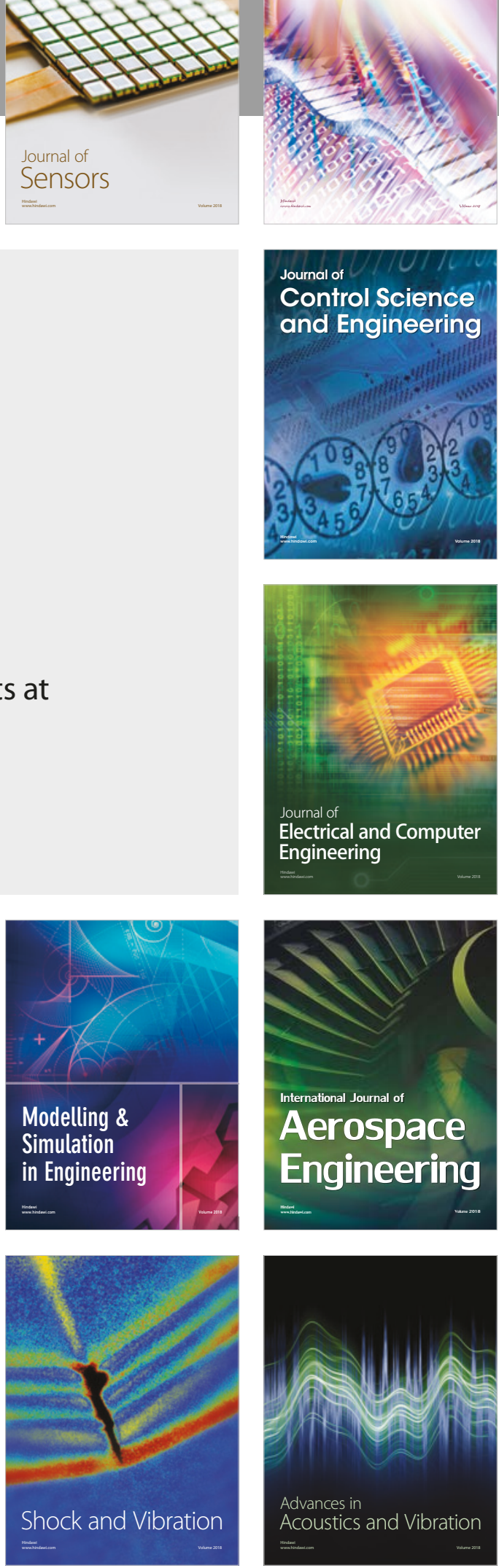